\title{
Interkulturelle Medienwissenschaft und Kulturkonflikt
}

\author{
Ernest W. B. Hess-Lüttich (Bern)
}

\begin{abstract}
The paper attempts a combination of intercultural studies and media studies. It first provides a terminological framework for the analysis of intercultural communication. Secondly, it gives a short outline of current research in the field of intercultural media studies, with special reference to the press and to television. It then opens up a perspective of empirical investigation into forms and functions of the depiction of ethnic minorities in the media, of implicit racism and hidden forms of discrimination in the news broadcasting, and of the image of foreigners in contemporary film. The main part of the paper is, on the one hand, devoted to the observation of the presence of foreigners in German TV programmes and the ways they are treated in with in various formats of broadcasting. On the other hand, it takes a closer look at the role of media for ethnic minorities, whether or not they serve a policy of integration and how the minorities make use of their own newspapers and television programmes. In a concluding paragraph a new field of research within intercultural media studies is outlined: development communication and eco-semiotics. Here, intercultural, institutional, and interpersonal communication processes merge in sustainable instruction procedures for the implementation of knowledge relevant for technology, environment and health in so-called low income countries.
\end{abstract}

\section{$1 \quad$ Interkulturelle Kommunikation}

Kulturelle Differenz, transkultureller Kontakt, Interkulturalität gelten vielen Anthropologen als Grundbedingung zivilisatorischer Entwicklung. Auf Interkulturelle Kommunikation richtet sich das Interesse zahlreicher Disziplinen, die ihren gemeinsamen Gegenstand je fachspezifisch explizieren, fachterminologisch definieren, fachhistorisch konturieren. In der Begegnung zwischen Angehörigen verschiedener Kulturen wird das Medium ihrer Verständigung problematisch, insofern die Regeln seines Gebrauchs wechselseitig in Frage stehen. Gegenseitiges Verstehen kann dadurch beeinträchtigt, aber auch bereichert werden. Indem etwa das automatische Routinehandeln im alltäglichen Gespräch durch die Konfrontation mit anderen, 'fremden' Routinen desautomatisiert wird, werden seine Strukturen und Prozesse, Muster und Schemata, Zeichen-Einheiten und Verknüpfungsregeln schärfer ins eigene Bewußtsein gehoben (Gumperz 1982). Die Erforschung Interkultureller Kommunikation in ihren alläglichen wie ästhetischen, historischen, materialen und institutionellen Aspekten kann im Zeichen global zunehmender transkultureller Kontakte, 
Kontexte, Konflikte auch in einer sozial- und kulturwissenschaftlich fundierten Medienwissenschaft nur an Bedeutung gewinnen (Hess-Lüttich ed. 1992; id. et al. eds. 1996).

Das Wort Interkulturalität ist eine zusammengesetzte Ableitung aus dem Präfix inter- (< lat. inter $=$ unter, zwischen $)$ und dem Nomen Kultur $(<$ lat. cultura $=$ Landbau, Pflege [des Körpers und des Geistes]). Die von Cicero eingeführte Metapher wird im Deutschen erst im Späthumanismus (Pufendorf) wiederaufgenommen und zunächst parallel gebraucht für Feldbau und Bodenbewirtschaftung einerseits, für die Pflege der geistigen Güter (cultura animi) andererseits. Aus der zweiten Bedeutung erwächst (seit Herder) der allgemeinere Begriff von Kultur als Bezeichnung für die Gesamtheit der geistigen und artistischen Leistungen einer Gemeinschaft, die für die Ausbildung ihrer Identität als sozialer Gruppe (politischer Nation, sprachlicher Gemeinschaft etc.) konstitutiv angesehen werden. W. v. Humboldt stellt ihn (folgenreich) dem der Zivilisation gegenüber. Tyler (1871) sucht "that complex whole which includes knowledge, belief, art, morals, law, custom, and any other capabilities and habits acquired by man as a member of society" mit dem Begriff zu erfassen, der seither in angelsächsischer Tradition auf die Gruppe (community) bezogen wird: "A culture refers to the distinctive way of life of a group of people, their designs of living" (Kroeber/Kluckhohn 1952: 86). Die Phänomenologie der Lebenswelt von Individuen als sozialen Subjekten in kulturell definierten sozialen Systemen (Alfred Schütz) führt zur akademischen Institutionalisierung der cultural studies, die wiederum seit den 1960er Jahren für die liberale Programmatik der Philologien in den zunehmend als multikulturell dargestellten Vereinigten Staaten von Amerika (new ethnicity) konzeptualisierende Funktion gewinnen und in den 1970er Jahren zur Etablierung des Lehr- und Forschungsgebiets der Intercultural Studies beitragen (Hansen 1995).

Interkulturelle Kommunikation im weiteren Sinne (Hess-Lüttich 1989; Ehlich 1996; Maletzke 1996; Jandt 1998) ist ein intra-nationales wie inter-nationales Alltagsphänomen, dessen wissenschaftliche Beachtung im vierten Quartal des letzten Jahrhunderts stetig zugenommen hat, mit der Tendenz systematischer Gebietsbildung bis hin zur akademischen Institutionalisierung, wie sie sich in der Gründung von Zeitschriften, Buchreihen, Handbüchern, wissenschaftlichen Vereinigungen, Instituten und Lehrstühlen niederschlägt (Rehbein ed. 1985; Redder/Rehbein eds. 1987; Jandt 1998; Wierlacher ed. 2000).

Die medienwissenschaftliche Thematisierung von Interkulturalität widmet sich heute im transdisziplinären Dialog mit Linguisten, Ethnologen, Soziologen, Pädagogen, Psychologen, Text- und Kommunikationswissenschaftlern einem weit gefächerten Aufgabenfeld: der Rolle der Sprache in interkulturellen (interpersonalen wie intermediären) Kontakten und Kontexten, der Verständigung mit oder zwischen Ausländern, der Fremdheitswahrnehmung in den Medien und ihrer xenologischen Untersuchung, dem Potential an Mißverständnissen und den Ursachen von Konflikten in interethnischer Kommunikation, den Problemen interkulturellen Lernens und Übersetzens in und mittels Medien, dem Verhältnis von Minderheitskulturen (Subkulturen) und Mehrheitskulturen im Mediensystem bzw. im Mediengebrauch, der Entwicklung von Aus- und Weiterbildungsmaßnahmen zur Vorbereitung auf Tätigkeiten im Ausland (z.B. Entwicklungskommunikation) bzw. zur Vermittlung interkulturellen Wissens 
im Inland (z.B. im Bereich Deutsch als Zweit- und Fremdsprache), der kulturspezifischen Prägung der Fach- und Wissenschaftskommunikation bzw. der interkulturellen Kommunikation am Arbeitsplatz in multinationalen Unternehmen, den Funktionen und Wirkungen der Medien der schönen Künste (Literatur, Theater, Film) in der Vermittlung zwischen den Kulturen, der interkulturellen Kommunikation in und durch Presse, Funk, Fernsehen sowie den Auswirkungen moderner Technologien auf die internationale Kommunikation. Neue Aufgaben wachsen der interkulturellen Medienwissenschaft zu durch die Einbeziehung komparatistischer, theater-, film- und fernsehwissenschaftlicher Fragestellungen vor allem im Bereich der Stoff-, Motiv-, Einfluß- und Konfliktforschung (Krusche 1985; Günthner 1993; Rauchfleisch ed. 1994; Fischer-Lichte et al. eds. 1995; HessLüttich ed. 1992) sowie in der Konzeption einer interkulturellen Medien-Geschichte (Großklaus [2003]; Faulstich [1997 ff.]). In diesem Zusammenhang wird auch gefordert, das Fach als "Medienkulturwissenschaft" neu zu konzipieren (Schmidt 1996) und insgesamt für interkulturelle Perspektiven zu öffnen.

\section{Interkulturelle Medienkommunikation}

In dem rasant expandierenden Sektor der kritischen Diskursanalyse kontemporärer Medienkommunikation bildet die Untersuchung von Formen und Funktionen der Diskriminierung von Minderheiten in den Medien bislang noch ein vergleichsweise schmales Segment. Empirische Analysen im Schnittfeld von Kultur-, Kommunikations- und Medienwissenschaften, Sprach-, Literatur- und Sozialwissenschaften, Psychologie und Semiotik müßten hier das Terrain genauer ausleuchten. Bisher lag der Schwerpunkt solcher Ansätze vor allem im Bereich der Rassismuskritik auf der Grundlage der (publizistikwissenschaftlichen, textlinguistischen, diskursanalytischen) Untersuchung von Pressetexten (Merten/Ruhrmann et al. 1986; van Dijk 1993; Jäger 1997a; Ruhrmann 1999). Inhaltsanalytische Arbeiten haben das Bild der Ausländerin der Presse herausgearbeitet (Küpfer 1994), die Asylberichterstattung kritisch unter die Lupe genommen (Hömberg/ Schlemmer 1995), Stereotypen und Metaphern auf ihren latent xenophoben Inhalt hin geprüft (Wagner 2000) und gefragt, "inwieweit einzelne Bezeichnungen (und Sprachregelungen) auf die öffentliche Meinung vorurteilsauslösend wirken" (Ruhrmann 1997: 63).

Funk und Fernsehen sind (nach angelsächsischem Vorbild: cf. Dines/Humez eds. 1995) im deutschsprachigen Raum erst seit Mitte der 90er Jahre Gegenstand systematischer Aufmerksamkeit im Hinblick auf das Thema Ausländer, Fremde, Migration und Diskriminierung geworden. Zwar gibt es im Hörfunkbereich nicht wenige kritische Hinweise auf bestehende Ausländerprogramme und Initiativen zu deren Verbesserung (cf. Hess-Lüttich 1992; Meier-Braun/Kilgus 1998), "systematische Analysen der Berichterstattung über Ausländer blieben jedoch unbekannt" (Ruhrmann 1997: 59).

Im Bereich des Fernsehens konzentrierte sich die Medienforschung auf Fremdbilder in der Berichterstattung (Eckardt/Horn 1995) oder den Zusammenhang zwischen fremdenfeindlichen Schlüsselereignissen und Nachahmungstaten (Brosius/Esser 1995) und Defizite in der Behandlung von Themen wie Rassismus, Rechtsextremismus, Ausländer und 
Asyl (Funk/Weiß 1995). Im engeren Bezirk der Germanistik und Linguistik ist es vor allem das Verdienst von Gruppen um Ruth Wodak in Wien, Georg Stötzel in Düsseldorf, Siegfried Jäger in Duisburg und Harald Burger in Zürich, das Thema Migration und Medien, öffentliche Kommunikation und Fremdverstehen mit Nachdruck auf die Agenda gesetzt zu haben. Die von diesen Gruppen vorgelegten Arbeiten über die medientypischen "Formen von rassistischen Diskursen" (Matouschek et al. 1995), das "Reden über Ausländer" in den Medien (Jung et al. eds. 1997) oder deren vorurteilsverstärkende "Kollektivsymbolsysteme" (Link 1982), über "Rassismus und Medien" (Jäger/Link eds. 1993) oder "Gewalt im Gespräch" der TV-Talkshows (Luginbühl 1999) seien hier nur stellvertretend genannt für den durch zahlreiche Studien bereiteten Boden, auf dem das Thema in den Medienwissenschaften, aber auch innerhalb der Germanistik und Linguistik, fruchtbar weiterverfolgt werden könnte.

So gilt es vor allem, die empirische Basis der Studien zu verbreitern und die medienwissenschaftliche Analyse besser mit den inhaltlichen und kontextuellen Ebenen zu verknüpfen. Darüber hinaus sind die dialogischen Strukturen genauer zu untersuchen, in denen Fremdheit als Beziehungsmodus generiert wird. Dabei sind auch die indirekten symbolischen Mechanismen und die nichtsprachlichen Ebenen einzubeziehen, die als ritualisierte Formen der Grenzziehung figurieren und damit für die Definition des Kommunikationsverhältnisses und die Regulation des emotionalen Interaktionsklimas zentrale Bedeutung gewinnen.

Dies hat sich etwa Tanja Thomas (in Vorb.) vorgenommen, wenn sie in ihrem Promotionsvorhaben der Frage nachgehen will, wie sich Medienschaffende in Fernsehgesprächen gleichsam ungewollt, d.h. entgegen ihrer eigenen Absicht, durch ihre Sprache und ihren medienspezifisch inszenierten Umgang mit Ausländern an deren Diskriminierung beteiligen. Dabei geht es ihr weniger um den Aufweis rassistischer Redeweisen in Terminologie und Metaphorik als um die impliziten, mehr oder weniger verborgenen Mechanismen der Diskriminierung, wie sie sich zeigen in der Themenwahl (z.B. als Polarisierung in der Talkshow "Kerner": "Ausländer sind die besseren Männer"), in der "Skandalinszenierung" (z.B. als Täter-Opferrollenkonstruktion in der Talkshow "Vera am Mittag": "Ehe mit einem [muslimischen, gewalttätigen] Ausländer"), in der Reproduktion von Stereotypen (z.B. als Klischee-Verstärkung in der Talkshow "Bärbel Schäfer": "Was reizt dich nur an farbigen Männern?"), in der Personalisierung von Interpretationsmustern (z.B. in der Darstellung binationaler Beziehungen als "Problem" in der Talkshow "Bärbel Schäfer": "Sie konnten zusammen nicht kommen. Sabine liebt Mehmet"), in der Herstellung vereinfachender Kausalbezüge (z.B. zwischen Kriminalität und Ausländern) und in der Emotionalisierung der politischen Debatte über Migration und Integration (z.B. in Erich Böhmes "Talk im Turm": "Doppelte Staatsbürgerschaft?").

Angesichts des Spektrums, das diese Untersuchungen in der jüngsten Zeit zur Diskriminierung im Diskurs der Medien Presse, Funk und Fernsehen entfaltet haben, überrascht die Lücke einschlägiger Fragestellungen - wenn man vom US-Kino einmal absieht (cf. Scharenberg 1999) - in Bezug auf das Medium 'Film'. Diese Lücke (und damit das Desiderat ihrer Schließung) konstatiert zumindest eine programmatische Skizze am Beispiel 
eines rassismuskritischen Kurzfilms (Pepe Danquart: "Schwarzfahrer") und eines Dokumentarfilms (Peter von Gunten: "They teach us how to be happy"), der die Praxis der institutionellen Befragung von Asylbewerbern aufs Korn nimmt (Hess-Lüttich 2001). In Hollywood wurde T. Coraghessan Boyles Roman The Tortilla Curtain (dt. América) verfilmt als Parabel über die Migranten aus Mexiko. Von illegalen Immigranten handelt auch der Film "Brothers in Trouble", in dem der in England aufgewachsene Inder Udayan Prasad den konfliktträchtigen Alltag in Londoner Quartieren zeigt, in denen die Einwanderer vom indisch-pakistanischen Subkontinent schon die Mehrheit der Bevölkerung stellen. "Harte Unterhaltung" nach Art der Briten ("Mein wunderbarer Waschsalon") bieten auch die jungen deutsch-türkischen Filmemacher wie Neco Çelik, der neben seinem Job als Sozialarbeiter im Berliner Jugendtreff Naunynritze einen Film für den WDR dreht. Alltag der Berlin-Türken ist das Thema der Filme von Thomas Arslan ("Dealer") oder Yüksel Yavuz ("Aprilkinder"). Der Regisseur Kutlug Ataman, der nach etlichen Jahren in Berlin, aber auch in Paris und Los Angeles, heute wieder in Istanbul arbeitet, bietet mit seinem Film "Lola und Bilidikid" eine multikulturelle Mixtur aus türkischem Melodram, deutscher Familiengeschichte und amerikanischem Thriller, aber auch die anrührende Liebesgeschichte zweier Männer im Kiez und rührt damit an das Tabuthema der Homosexualität im Islam, dessen subtile Darstellung im Medium Film eine interkulturelle Herausforderung eigener Art darstellt.

Das Thema Inter- oder Multikulturalität scheint seit kurzem bei Filmemachern und Medienleuten, aber auch bei einem Teil des Publikums, auf so lebhaftes Interesse zu stoßen, daß das Amsterdamer Dokumentarfilm-Festival ihm mit einer eigenen neuen Reihe unter dem Titel "Global Motion" ein vielbeachtetes Forum bietet für Filme über Flüchtlinge, Migranten, Asylanten. Im österreichischen Linz haben Sabine Derflinger und Bernhard Pötscher ihren Dokumentarfilm "Achtung Staatsgrenze!" in einem Abschiebungsgefängnis gedreht. Maurizio Zaccaro schildert in seinem Film "Articolo 2" die vergeblichen Versuche des Algeriers Mohamed, seine "zweite" Familie bei sich in Italien aufzunehmen, in der sein Status freilich als Bigamie verfolgt wird. Der Film "Winterblume" des in Köln lebenden Kadir Sözen beschreibt in eindringlichen Bildern den illegalen Versuch eines abgeschobenen Türken, zu seiner Familie zurückzukehren. Mehmet Umut erfriert im Schnee eines Alpenpasses, während das Gericht das Urteil fällt, seine Rückkehr doch noch zu erlauben. Meist aber steht am Ende des Verfahrens, nach Jahren zermürbenden Wartens unter Fremden in Asylunterkünften, mit fremder Kost und bei strengem Arbeitsverbot, die Abschiebung, die in Österreich 'Schubhaft' heißt und in der Schweiz 'Ausschaffung'. Die wachsende Zahl von interkulturellen Spielfilmen kann dazu motivieren, den Blick noch genauer auf die nicht immer unproblematischen Formen der Vergesellschaftung des Migrationsproblems nicht nur in der Presse und in den Institutionen, sondern auch in der (multimedialen) Unterhaltungsindustrie zu lenken (Hess-Lüttich 1997). 


\section{$3 \quad$ Fremde in den Medien}

Die Entdeckung des Themas "Medienberichterstattung über Ausländer" datiert Ruhrmann (1999: 96) in die 50er Jahre, als in den USA die Frage nach der Medienrepräsentanz von (ethnischen) Minoritäten untersucht wurde. Freilich waren diese Minoritäten in aller Regel Amerikaner, also nicht Ausländer. Im deutschsprachigen Raum taucht das Thema als Aufgabe der Medienwissenschaft erst in den 80er Jahren auf, als im Fernsehen zunehmend Fremde ins Bild rückten. Man erinnert sich an Filme von Gertrud Pinkus ("Das höchste Gut der Frau ist ihr Schweigen"), Tevfik Baser ("40 qm Deutschland"), Werner Schroeter ("Palermo/ Wolfsburg"); an Fernsehspiele wie "Aischa" oder "Tu was, Kanake" mit ihrer Psychopathographie einer in der Fremde ausgesetzten Existenz; an plastische Milieustudien in Familien- und Krimiserien wie "Lindenstraße" und "Tatort"; an Spielserien über das Zusammenleben von Deutschen und Ausländern ("Unsere Nachbarn - die Baltas", "4 Hoffmanns und 5 Cupovics"); an zahllose Talkshows und Fernsehdiskussionen über Ausländerprobleme oder Asylrechtsfragen; an Kulturmagazine mit Beiträgen über hier schreibende "Gastarbeiter" und Bücher über den Alltag der Ausländer; an etliche Features und Dokumentarsendungen über Ausländerfragen ("Kiel Gaarden - ein Stadtteil lebt mit seinen Ausländern"; "Herr Ugurlu schlachtet einen Hammel"; "Die Rückkehr der Familie Arzik"; "Die Serefs, die Konuralfs und wir"; "Knoblauch, Kölsch und Edelweiß"; "Ausländer raus ein Ort in Deutschland: Rheda-Wiedenbrück").

Aber die Erinnerung täuscht: der Anteil solcher Sendungen am Gesamtprogramm war eher gering: nach einer in der Mitte der 80er Jahre durchgeführten Untersuchung (Kühne-Scholand 1987: 81) erreichte er in einer Stichprobe von ca. 460 Programmstunden gerade 4,5 \% der Sendezeit (inkl. aller Beiträge innerhalb von Sendeplätzen, also auch der Kurzbeiträge mit einer Durchschnittslänge von $1^{1 / 4}$ Minuten in Nachrichtensendungen, Magazinen, Diskussionen). Dabei handelte es sich überwiegend (d.h. zu ca. $80 \%$ ) um ereignisbezogene Beiträge der aktuellen Berichterstattung, fast nie aus der Perspektive oder im Interesse der Betroffenen. Nach der zeitgenössischen Diagnose von Hans Janke (1987: 62) vom AdolfGrimme-Institut waren die "Informationsangebot[e] dürftig, [...] tiefergehende Beschreibungen und Analysen eher rar."

Diese Diagnose traf weitgehend auch auf die Spielfilme zu. Zwar bemühte sich die ARD, mit guten Filmen aus Ländern der Dritten Welt (z.B. in der Reihe 'der Studio-Film') über Seh- und Erzählgewohnheiten des europäisch-amerikanischen Kinos hinauszugehen. Aber manche dieser Filme wirkten eher wie bebilderter Erdkundeunterricht denn als Vehikel oder Impuls interkultureller Verständigung über ästhetische Texte mit Sedimenten außerästhetischer Erfahrungen. Dabei kommt gerade dem Fernsehen im Hinblick auf interkulturelle Kommunikation besondere Bedeutung zu als Medium der Begegnung mit Spielfilmen aus fremden Kulturkreisen für einen größeren Rezipientenkreis. Dieser Verantwortung suchen die öffentlich-rechtlichen Sender in bescheidenem Umfang Rechnung zu tragen, während die privaten Fernsehanbieter Ausländerthemen nach wie vor in eher oberflächlicher Manier präsentieren (Eckhardt/Horn 1995: 10; Ruhrmann 1999: 101). 
Die Diskrepanz zwischen der Präsenz fremder Kulturen in Magazinen und Nachrichten und der Zahl von Spielfilmen ist immer noch markant. Vor dem Hintergrund der jahrhundertelangen europäischen Tradition der ebenso intensiven wie konflikthaften kulturellen Auseinandersetzung mit dem Vorderen Orient ist das Defizit an arabischen Filmen etwa fast noch gravierender als das immer noch sehr bescheidene Angebot von Filmen aus dem afrikanischen oder südostasiatischen Raum, denn anders als bei diesen Filmen ist bei solchen aus dem arabisch-islamischen Kulturkreis ein historisch abgelagertes Vorwissen in Rechnung zu stellen, das sich in Form stereotyper Muster als Wahrnehmungsfilter erweist, der umgekehrt in deutschen Produktionen nicht selten noch verstärkt wird durch Klischeevorstellungen in Serien (z.B. in "Tatort"-Produktionen wie "Duisburg-Report" von Hajo Gries oder "Ein Schuß zuviel" von Wolfgang Mühlbauer) und Magazin-Sendungen (wie "SOS für So 36" über Gastarbeiter in Kreuzberg).

Die inneren Strukturen der fremdkulturellen Gemeinschaft bleiben verborgen, Maßstäbe der Residenzgesellschaft werden von außen angelegt, vereinfachende Monokausalismen konstruiert und vorschnelle Wertungen vorgenommen. Der verräterische Metaphernschatz der vox populi und ihrer politischen Verstärker wird zuweilen unbekümmert übernommen in der Rede über "Die große Flut" (Sendung von Heinz Hemming/Bernhard Töpper, ZDF 21.8.1986) bis zu den Titelgeschichten des SPIEGEL (z.B. "Gefährlich fremd" v. 14.4.1997 oder "Zu viele Ausländer?" v. 23.11.1998) über den "Sprengsatz" der Ausländerzahlen, deren Zunahme als "Zeitbombe" wirke (cf. die Medienanalyse dazu von Sarigöz 1999), und der populistischen Übernahme von Varianten der "Das Boot ist voll"-Metaphorik durch den deutschen Innenminister Otto Schily in der Asyl- und Migrationsdebatte bis Ende der 90er Jahre. (Erst ab 2000 kündigt sich ein Umdenken an, weil die Wirtschaft aus demographischen Gründen wieder Arbeitskräfte aus dem Ausland nachzufragen beginnt: cf. die nicht sehr erfolgreiche Greencard-Kampagne).

Das Fremde bleibt fremd und wird - zumal in Magazinen und Boulevardformaten - als Exotikon geboten. Je vertrauter der kulturelle Hintergrund (EU, USA), desto positiver das Fremdbild bei den deutschen Zuschauern. Islamische Kulturen bleiben den Befragten fremd (Eckhardt/Horn 1995: 2-10). Entsprechend mißtrauisch reagieren die Betroffenen. Türkische Spielfilme im ZDF riefen heftige Proteste seitens der Türken hervor, die Zeitung TERCÜMAN forderte das Ende der "Propaganda gegen die Türkei". Ähnliche Reaktionen gab es auf die gewiß gut gemeinte Ausstrahlung einer WDR-Serie von Güney-Filmen (Originalton mit Untertiteln) in den Dritten Programmen. In den Nachrichtensendungen kommen ethnische Minoritäten, wenn überhaupt, vornehmlich als Problemfälle ins Bild. Dies gilt erst recht für die aktuelle Berichterstattung im Hörfunk und in der Presse.

Sprach- und Inhaltsanalysen können einige der Ursachen der überwiegend negativen Wirkung solcher Meldungen und Informationen über die Ausländer freilegen. Zum Fernsehen liegen bis auf die Skizze von Kühne-Scholand (1987) erst seit der zweiten Hälfte der 90er Jahre systematische Studien zum Bild der Ausländer in diesem Leitmedium vor (Brosius/Esser 1995; Funk/Weiß 1995; Schatz et al. eds. 2000). Die Themen der Beiträge beziehen sich danach in erster Linie auf Asylsuchende und Flüchtlinge. Ihre Kultur und Geschichte, ihre 
Feste und Feiern kommen nur im Ausnahmefall vor. Möglichkeiten der Lösung ihrer Probleme werden in Maßnahmen der Legislative und Exekutive gesehen; andere Aspekte wie Verbesserung der Integrationshilfen und der Verständigung mit ihnen, ihre Selbstdarstellung und Interessenvertretung werden kaum genannt. Sie sind, zu $80 \%$, Objekt einer ereignisbezogenen Darstellung in problemorientierter Berichterstattung des Fernsehens. Sie kommen als Gruppe ins Bild, meist im Gedränge vor Meldestellen oder in engen Unterkünften. Dem deutschen Zuschauer erscheinen sie als Verursacher von Problemen, deren Folgen auch ihn belasten oder bedrohen (Kosten, Wohnungsnot, Arbeitsplatzkonkurrenz, "Überfremdung", Kriminalität usw. sind die thematischen Assoziationen). Die "Folgen weltweiter Migrationsprozesse und das Entstehen multikultureller Tendenzen werden in einer Semantik der Gefahren präsentiert" (Ruhrmann 1999: 102).

Die Printmedien sind im deutschsprachigen Raum erst seit Mitte der 80er Jahre Gegenstand genauerer Analysen im Hinblick auf das darin aufscheinende Bild der Ausländer. Besondere Bedeutung haben dabei die empirischen Studien von Merten, Ruhrmann et al. (1986) zur inhaltsanalytischen Auswertung eines repräsentativen Samples der bundesdeutschen Presse und Ruhrmann/Kollmer (1987) zur Berichterstattung über Ausländer in zwei Bielefelder Tageszeitungen erlangt. Sie fanden ausländische Arbeitnehmer in zwei Dritteln aller wertenden Artikel negativ dargestellt (64,8\%), Asylbewerber sogar in mehr als drei Vierteln aller Fälle (75,8 \%). Positive Bewertungen von Ausländern bezogen sich zu 80,6 \% auf Gäste, Künstler und Sportler (Merten et al. 1986: 90 ff.; Merten 1987: 71). Ausländer aus Südeuropa kommen dabei besser weg als solche aus der Türkei, Afrika oder Südostasien. Beim Vergleich positiver und negativer Bewertungen von türkischen und nicht-türkischen Arbeitnehmern in der deutschen Presse fand Merten (1987: 73) bemerkenswerte Unterschiede: das Saldo positiver minus negativer Bewertungen betrug bei den Griechen, Italienern, Spaniern usw. $+4,2 \%$, bei den Türken -41,6 \%. (Zur Entwicklung deutschtürkischer Medienbeziehungen s. Becker 2000).

Gegenüber dem Fernsehen kommt hinzu, daß die Presse im lokalen Bereich nahezu ein Monopol auf Informationen über Ausländer hat. Lokalberichterstattung gilt als Ort überwiegend positiver Nachrichten über das Vereinsleben, Sport, Einweihungen, Jubiläen usw. Nachrichten über Ausländer finden sich darin selten; sie kommen vielmehr im Polizeibericht vor, dessen Hinweise auf den Status 'Ausländer' (Name, Nationalität, Hautfarbe, Akzent u.ä.) vom Lokaljournalisten sorgsam übernommen werden. Bei deutschen Tätern fehlen meist solche ethnischen Hinweise. Trotz zunehmender Sensibilisierung für das Dilemma der Journalisten, die über Ausländerkriminalität zu berichten haben, hat sich nach jüngsten Untersuchungen an diesen Befunden noch immer nicht viel geändert (Jäger, M. 1999; Jäger, S. 2000).

Je größer die kulturelle Distanz zwischen dem Einheimischen und der jeweiligen ethnischen Gruppe, desto geringer im Durchschnitt sein Kontakt zu den ihr angehörenden Individuen. Sein Wissen über sie bezieht er nicht aus direkter Interaktion, sondern aus den Medien. Dort aber erscheinen sie in negativer Verzerrung. Interkulturelle Kommunikation mittels Medien 
wird so, wenn nicht verhindert, so doch erheblich erschwert. Dies führt nach Merten (1987: 77) $\mathrm{zu}$ dem paradoxen Effekt, daß eine formal korrekte Berichterstattung eine Medienwirklichkeit erzeugt, "die zwar mit der realen Wirklichkeit nicht übereinstimmt, die aber gerade deswegen die reale Wirklichkeit im Sinne der Medienwirklichkeit verändert: Ausländer sind krimineller, fauler, schlechter als Einheimische - zumindest in den Köpfen der zeitungslesenden Durchschnittsbürger". Wirksam ist nicht die Wirklichkeit an sich, wie man weiß, sondern ihre Wahrnehmung, die Vorstellung präselegierter Wirklichkeit durch das autopoietische System der Medien (Luhmann 1996).

\section{$4 \quad$ Medien für die Fremden}

Wenn die Massenmedien die deutschen Adressaten zur interkulturellen Kommunikation schon nicht ermutigen, wie steht es umgekehrt mit dem Medienangebot für die ausländischen Mitbürger? Und wie nutzen sie es? Was hat sich geändert seit den ersten Studien über diese Fragen, die bereits Mitte der 80er Jahre in einer von ARD und ZDF initiierten Studie untersucht wurden, die ein recht genaues Bild darüber ergab, welchen Zugang Griechen, Italiener, Spanier, Türken in der Bundesrepublik der 80er Jahre zu solchen Medien gehabt haben, wie sie sie nutzten, was sie von ihnen erwarteten, wie sie sie bewerteten (Darkow et al. 1985; hier kurz resumiert nach Eckhardt 1987; cf. id. 2000).

Fernsehen und Hörfunk wurden danach von den Ausländern im Durchschnitt zunächst weit weniger genutzt als von den Deutschen - aus Zeitgründen: sie hatten weniger Freizeit dafür zur Verfügung. Die nicht-berufstätigen Frauen sahen überdurchschnittlich viel fern, desgleichen ihre Kinder im Vergleich zu ihren deutschen Altersgenossen, die jungen Männer hörten mehr Radio, Schallplatten und Cassetten (was heute kaum noch eine Rolle spielt im täglichen Medienkonsum der Jüngeren). Nicht überraschend: je besser die Sprachkenntnisse, desto intensiver die Nutzung der deutschsprachigen Medien. Das galt auch für die Zeitungen und Zeitschriften: nur etwas mehr als die Hälfte der Ausländer las die deutschsprachige Presse, am wenigsten die Türken (s.u.). Dem korrespondierte die Nutzung der speziellen TVund Radio-Sendungen für Ausländer: sie wurden am eifrigsten von den Türken, am wenigsten von den Italienern verfolgt. Sie wurden als 'Brücke zur Heimat' aufgefaßt, weniger als Hilfe zur Orientierung im Gastland. Insgesamt war die Bindung an diese speziellen Programme, die der Aufrechterhaltung der kulturellen Identität dienen sollten, eher gering, außer bei den älteren Migranten, vor allem solchen türkischer Herkunft.

Gerade diese speziellen Integrationsprogramme des Fernsehens wurden freilich in einigen Dissertationen und Magisterarbeiten mittlerweile einer ebenso detaillierten wie herben Kritik unterzogen. Das ZDF-Programm "Nachbarn in Europa", das sich an Jugoslawen, Griechen und Spanier, an Italiener, Portugiesen und Türken wandte, lieferte in 35 Minuten eines dreiviertelstündigen Programms überwiegend Folklore und Unterhaltungsmusik, auch Nachrichten aus der Heimat, in 14-täglichem Abstand. Deutsche Zuschauer wurden kaum erreicht, die ausländischen Zielgruppen waren in der Redaktion nicht gleichberechtigt vertreten, das eigene Ziel der 'Orientierungshilfe' und 'Integration' wurde verfehlt, bilanziert 
Michael Koschinski (1986: 129) in seiner Untersuchung interkultureller Kommunikationsprobleme der türkischen Minderheit.

Die Sendung des WDR "Ihre Heimat - unsere Heimat" kommt in seiner Untersuchung etwas besser weg; sie sei gegenüber der ZDF-Sendung 'politischer' gewesen, habe mehr Nachrichten aus dem Herkunftsland enthalten, nicht nur offizielle Verlautbarungen, auch Gegenstimmen. In der Redaktion waren kompetente Ausländer vertreten, vertieft durch persönliche Kontakte zwischen Sender und Vertretern der Minoritäten außerhalb, die manches auch ohne Rechtsanspruch auf Partizipation möglich machten. Dennoch sei auch hier die Nutzungshäufigkeit durch die Zielgruppen zurückgegangen, die Skepsis in der Beurteilung der Sendungen durch die Ausländer habe überwogen. Die Statistiken und Resultate der empirischen Erhebungen seither sprechen eine recht eindeutige Sprache, und zwar (trotz einzelner Differenzierungen) weitgehend unabhängig von der Nationalität der Zielgruppe, am ausgeprägtesten noch immer bei der türkischen Minderheit (Güntürk 1999).

Das Kommunikationsverhältnis bleibt asymmetrisch: Informationen, Handlungsmuster, Normorientierungen werden ohne Rücksicht auf den spezifischen kulturellen Hintergrund der Rezipienten von deutschen Redakteuren an die Minoritäten weitergegeben. Der potentielle Kultur-Transfer in umgekehrter Richtung wird nicht einmal erwogen, weil er der Intention der Integration in die Residenzgesellschaft zuwiderliefe. Daß diese freilich bislang, bei der sogenannten 'ersten' Generation zumindest, kaum gelungen scheint, belegt schon die Existenz solcher Sonderprogramme und ihre Trennung vom 'normalen' Programm. Das AdolfGrimme-Institut hat dem mit seiner Initiative "Mehr Farbe in die Medien" Ende der 90er Jahre Rechnung zu tragen versucht.

Schon Anfang der 80er Jahre hatte es mit dem Medienverbundprojekt "Ausländer-Inländer" ein Zeichen gesetzt, das laut Presseinformation des Instituts (AGI ed. 1983) durch die 'multimediale' Konfrontation von Spielfilmen, Dokumentarsendungen, Seminaren, Presseserien, und Veranstaltungen versuchte, "von Fernsehprogrammen ausgelöste emotionale Betroffenheit als Motivationsbasis für [interkulturelle] WeiterbildungsVeranstaltungen" zu nutzen. Das Ergebnis war allerdings nach der zitierten Untersuchung von Koschinski (1986: 110 f.) eher ein Beleg für die "mangelnde Kooperationsfähigkeit von wissenschaftlichem Institut und Sendeanstalt", es war nämlich insgesamt "nicht geeignet, integrationsfördernd zu wirken - weder bezogen auf die Residenzgesellschaft, noch bezogen auf die Minderheit". Fazit der kritischen Studie war seinerzeit, daß das "öffentlich-rechtliche Fernsehen [...] keinen befriedigenden Beitrag zur Lösung dieser interkulturellen Kommunikationsprobleme" leiste (Koschinski 1986: 126). Heute zeigt sich, daß die privaten Sender die 'Marktlücke' nicht genutzt haben; vielmehr scheint sich die Prognose von Hans Janke (1987: 65) zu bestätigen: "Rundfunkbeschäftigung mit Ausländern [...] wird auf absehbar lange Zeit öffentlich-rechtlich sein oder nicht sein".

Tatsächlich liegt der Anteil der Journalist(inn)en nicht-deutscher Herkunft noch immer weit unter dem Anteil von ca. $10 \%$ Ausländern in der Bevölkerung. Hier setzt nun das neue Modellprojekt des Adolf-Grimme-Instituts zur Qualifizierung von Migrant(inn)en im Journalismus an: Bewerber aus 13 Herkunftsländern wurden ausgewählt und für ihre künftige 
Tätigkeit in Funk und Fernsehen vorbereitet. Trotz ihrer besseren Berufsaussichten stehen aber auch sie vor dem Dilemma, leicht auf die Rolle des Spezialisten für Minderheiten festgelegt zu werden, abgesehen davon, daß nach der kritischen Diagnose von Jungk (1999: 225) die Bereitschaft der Sendeanstalten zu einer interkulturellen Öffnung noch immer nicht sehr ausgeprägt sei; deshalb stelle sich heute nachdrücklicher denn je "eine Aufgabe: die ständige Lobbyarbeit für die Notwendigkeit interkultureller Personalpolitik im Medienberiech, für die sich auch die Migrantinnen und Migranten stark machen müssen" (Jungk 1999: 227).

Aber auch andere Projekte der 80er Jahre haben die in sie gesetzten Erwartungen nicht erfüllt. Die Erfahrungen mit ausländerspezifischen Programmen in sog. Kabelpilotprojekten in Berlin, Dortmund oder Ludwigshafen klingen aus heutiger Sicht ziemlich ernüchternd. Trotz optimaler Bedingungen etwa in Berlin mit seiner hohen Ausländerdichte in bestimmten Wohnvierteln (mit entwickelter eigener Infrastruktur und hohen Nachfragepotentialen) hat dort kein kommerzieller Anbieter mit einem kohärenten Ausländerprogramm überlebt. Schon früh resümierte Otfried Jarren (1987: 108) für das Fernsehen lakonisch: "Ein Programm für die ausländischen Mitbürger gibt es nicht. Es existiert allenfalls ein relativ differenziertes Fernsehangebot für die türkischen Mitbürger."

Nachdem die Ausländer der ersten Generation durch die Medien also kaum erreicht werden konnten, widmet man daher der jeweiligen zweiten und dritten Generation größere Aufmerksamkeit, z.B. mit speziellen Integrationsprogrammen des Schulfernsehens. Hier ist die Kooperation zwischen Medienpraxis und Medienwissenschaft inzwischen recht weit gediehen, wenn auch das seinerzeit programmatisch verkündete Ziel der 'interaktionistischen Integration' noch kaum erreicht worden sein dürfte. Aber Programme wie "Treffpunkt Deutsch" für ausländische Kinder im Grundschulalter haben immerhin versucht, den Dialog zwischen den einheimischen und deutschen Kindern in Gang zu setzen. Damit lassen sich freilich nicht die Konflikte lösen, mit denen ausländische Kinder zwischen den Stühlen der kulturellen Prämissensysteme zu Hause und in der Schule immer noch täglich konfrontiert sind. Die extremen Brüche in Sozialisation und Spracherwerb führen bekanntlich nicht selten zu Defiziten der Kommunikationsfähigkeit in beiden Kulturen (mit entsprechenden in ihrer gesellschaftspolitischen Brisanz endlich erkannten Konsequenzen für den Schul- und späteren Berufserfolg).

Eigene Erfahrung und Fernsehrealität klaffen nicht selten in schwer überbrückbarer Weise auseinander, selbst bei den eigens für ausländische Kinder produzierten Sendungen wie der verdienstvollen NDR-Reihe "Treffpunkt Deutsch" oder der WDR-Reihe "Islam". Diese Reihe bot deutschen Kindern zwar viele Informationen über den weltanschaulich-religiösen Hintergrund ihrer türkischen Spielgefährten, war selbst aber auch nicht frei von ethnozentrischen Prämissen der Bildauswahl. Demgegenüber bot die Serie "Kinder in anderen Glaubensgemeinschaften" ein Beispiel für die positive Nutzung von Möglichkeiten der Kooperation zwischen Wissenschaft und Praxis zur wirksamen Verbindung von Medien und interkultureller Vermittlung: in dem Film über "Nazmiyes Kopftuch" wurden die Verständigungsprobleme zwischen deutschen und türkischen Kindern nicht beschönigt, 
sondern in einfacher Erzählstruktur anschaulich bewußt gemacht. Ein Gegengewicht zu dem überwiegend immer noch negativen Bild des Islam in den deutschsprachigen Medien bieten solche Versuche kaum: neue Untersuchungen belegen die weitgehende Gleichsetzung muslimischer Wertorientierung mit Repression, Mittelalter, Frauenunterdrückung, Fundamentalismus, Terrorismus; dichotomische Bilder verstärken bestehende Stereotypen vom vermeintlich friedlichen 'Westen' und stets gewaltbereiten Islam (Hafez 1999).

So kann kaum überraschen, daß sich gerade die größte islamische Minorität in Deutschland längst selbst ein differenziertes Medienangebot aufgebaut hat (Güntürk 1999; Becker 2000). Anfang der 70er Jahre erschienen erstmals in nennenswertem Umfang türkische Zeitungen, heute gibt es acht überregionale Tageszeitungen, einige ganz in Deutschland produzierte Wochenzeitungen und seit 2001 auch ein bikulturelles Magazin, das sich an junge DeutschTürken wendet. Parallel etablierte sich in den 80er Jahren ein umfangreicher Kino- und Videomarkt mit eigenen türkischen Verleihfirmen und Videotheken. Seit dem Beginn des Privatfernsehens in der Türkei Anfang der 90er Jahre werden auch in Deutschland zunehmend türkische Sender empfangen. In der zweiten Hälfte der 90er Jahre liest die Mehrheit der Türken in Deutschland (über $55 \%$ ) ausschließlich türkischsprachige Zeitungen, ca. $38 \%$ lesen sowohl deutsche als auch türkische Zeitungen, nur $6 \%$ lesen allein deutsche Zeitungen. Beim Fernsehkonsum ist es nicht anders: über die Hälfte der Türken verfolgen das Fernsehen (überwiegend die Programme der fünf wichtigsten türkischen Sender) mittlerweile mehr als drei Stunden am Tag; nur $7 \%$ sehen ausschließlich deutsche Programme.

Für einen Erfolg der bisherigen Bemühungen zur Integration dieser Minderheit sprechen die Zahlen nicht gerade. Sie sind vielmehr ein eher zwiespältiges Indiz eigenkultureller Emanzipation gegenüber einer unverständlich gebliebenen Umwelt, aber auch interkultureller Sprachlosigkeit: beunruhigender Ausdruck einer Tendenz zur Selbstisolierung, Reflex auf die Ghettosituation, Rückzug ins Vertraute. Heimatliche Komödien, Musikfilme und Melodramen sind das Geschäft türkisch geführter Verleihfirmen, ihre Werbeetats bei hiesigen türkischen Zeitungen steigen sprunghaft. Die desintegrierende Wirkung dieser Entwicklung läuft den interkulturellen Bestrebungen unseres Kultur- und Medienmanagements in einer nicht mehr zu verharmlosenden Weise zuwider. Wenn der Dialog zwischen den Subkulturen einer zunehmend multikulturellen Gesellschaft nicht abbrechen soll, dürfen wir die Alarmzeichen nicht ignorieren: eine Herausforderung auch für die interkulturelle Medienwissenschaft.

\section{$5 \quad$ Konflikt- und Entwicklungskommunikation}

Das Konfliktpotential dieser Entwicklungen ist mittlerweile sowohl von der Wissenschaft als auch von Teilen der Medien und der Politik erkannt. Immer mehr Studien widmen sich in jüngster Zeit dem Rassismus- und Migrationsdiskurs in den Medien (z.B. van Dijk 1993; Matouschek et al. 1995; Hess-Lüttich 1997; Ruhrmann 1997; Luchtenberg 1999: 133-168; Butterwegge et al. eds. 1999; Reisigl/Wodak 2000; id. eds. 2000; Schatz et al. eds. 2000). Im Zeichen der Globalisierungs- und der Multikulturalitätsdebatten eröffnet sich hier in der Tat ein weites Feld. Eine Parzelle darin ist freilich noch so gut wie gar nicht beackert: die 
Entwicklungskommunikation. Sie soll abschließend wenigstens als exemplarisches Stichwort genannt werden für die Formulierung weiterer künftiger Aufgaben einer interkulturellen Medienwissenschaft (neben vielen anderen, die in diesem Rahmen nicht behandelt werden können, wie z.B. die historisch-vergleichende Dimension, auf deren Bedeutung hier nur nachdrücklich verwiesen werden kann: s. hierzu Großklaus [im Druck]).

Während die bisher genannten Sektoren der interkulturellen Medienwissenschaft in den letzten beiden Dekaden im deutschsprachigen Raum einen unerhörten Aufschwung nahmen, tut sich im Bereich der sog. development communication (Entwicklungskommunikation und Öko-Semiotik) ein völlig neues Forschungsfeld auf. Hierbei geht es um die systematische Verbindung von Ergebnissen der Erforschung interkultureller, institutioneller und interpersoneller Kommunikation zum Zwecke der nachhaltigen Vermittlung technischen, umwelt- und gesundheitsrelevanten Wissens in Entwicklungsländern durch kontextspezifisch geeignete Medien.

Organisationen wie Amnesty International oder Greenpeace planen ihre Aufklärungskampagnen in den Medien heute supranational, aber das in den Industriestaaten damit für Fragen der Rechtssicherheit (als Bedingung wirtschaftlicher Investitionen) und nachhaltiger Ressourcenbewirtschaftung sensibilisierte öffentliche Bewußsein nützt wenig, wenn es in Ländern der Dritten Welt aufgrund mangelnden interkulturellen Wissens nicht zu vermitteln ist. Krisen und Konflikte haben dort ihre Ursachen häufig in einem schwer zu entwirrenden Geflecht von Problemen ökologischer Interessen und interkultureller Verständigung (zur Ökosemiotik allgemein Nöth 1996; zum Umweltdiskurs speziell Anderson 1997; Harré et al. 1998).

Am Beispiel der knappen Ressource Wasser konnte in einer Reihe von Fallstudien in Afrika gezeigt werden, wie solche Probleme erfolgreich gelöst werden können durch die Vermittlung technisch-ökologischen Wissens an die indigene Bevölkerung, deren Verhaltensänderung durch entsprechend geschulte einheimische Vertrauenspersonen und deren Einsatz mikrokulturspezifischer Lehrmaterialien bewirkt wurde (Genske/Hess-Lüttich 1999). Der interdisiplinäre Ansatz kombiniert Verfahren der discourse analysis und cultural studies und wendet sie an auf Instruktionsdiskurse in interkulturellen institutionellen settings (von der Mitarbeiterschulung internationaler Hilfsorganisationen bis zur face-to-face Interaktion lokaler mediators (bzw. animateurs) zwischen Entwicklungsingenieuren und Einheimischen in ariden periurbanen Regionen Ost- und Westafrikas). Die einzigartige Verbindung von Kommunikations- und Umweltwissenschaften, von Kultur- und Technikwissenschaften läßt schon heute das Potential erahnen, das daraus (exemplarisch) für die motivierende Verbindung der Erkenntnisfelder Medienkommunikation und interkulturelle Kommunikation erwachsen könnte.

\section{Literaturangaben}

Agr (ed.) (1983): Presseinformation Medienproduktverbund Ausländer - Inländer. Marl. AGI (ed.) (1998): Programmauftrag Interkulturelles Fernsehen (Tagungsreader). Marl. Anderson, Alison (1997): Media, culture and the environment. New Brunswick, NJ. 
Becker, Jörg (2000): "Türkisch-deutsche Medienbeziehungen: Phasen und Systematik". In: Schatz, Heribert et al. (eds.) (2000): 106-115.

Brosius, Hans-Bernd/Esser, Frank (1995): Eskalation durch Berichterstattung? Massenmedien und fremdenfeindliche Gewalt. Opladen.

Brünner, Gisela/Graefen, Gabriele (eds.) (1994): Texte und Diskurse. Methoden und Forschungsergebnisse der funktionalen Pragmatik. Opladen.

Brünner, Gisela/Fiehler, Reinhard/Kindt, Walther (eds.) (1999): Angewandte Diskursforschung, vol. 1: Grundlagen und Beispielanalysen. Opladen.

Bundeszentrale für Politische Bildung (ed.) (1987): Ausländer und Massenmedien. Bestandsaufnahme und Perspektiven. Bonn.

Butterwegge, Christoph (1999): "Massenmedien, Migrant(inn)en und Rassismus". In: Butterwegge, Christoph et al. (eds.) (1999): 64-89.

Butterwegge, Christoph/Hentges, Gudrun/Sarigös, Fatma (eds.) (1999): Medien und multikulturelle Gesellschaft. Opladen.

Darkow, Michael et al. (1985): Massenmedien und Ausländer in der Bundesrepublik Deutschland. Frankfurt/Berlin.

Dijk, Teun A. van (1993): Elite Discourse and Racism. Newbury Park.

Dijk, Teun A. van (ed.) (1997): Discourse Studies. A Multidisciplinary Introduction, vol. 2: Discourse as Social Interaction. London/Thousand Oaks/New Delhi.

Dines, Gail/Humez, Jean M. (eds.) (1995): Gender, Race, and Class in Media. A Text Reader. London/Thousand Oaks/New Delhi.

Eckhardt, Josef (1987): "Ausländer als Zielgruppe der Massenmedien". In: Bundeszentrale für Politische Bildung (ed.) (1987): 85-92.

Eckhardt, Josef (2000): "Mediennutzungsverhalten von Ausländern in Deutschland". In: Schatz, Heribert et al. (eds.) (2000): 265-271.

Eckardt, Josef/Horn, Imre (1995): "Fremde Kulturen im Fernsehen. Ergebnisse einer qualitativen ARD/ZDF-Grundlagenstudie". Media Perspektiven 1: 2-10.

Ehlich, Konrad (1996): "Interkulturelle Kommunikation". In: Goebl, Hans/Peter H. Nelde et al. (eds.) (1996): Kontaktlinguistik. Ein internationales Handbuch. Berlin/New York: 920931.

Fairclough, Norman/Wodak, Ruth (1997): "Critical Discourse Analysis". In: Dijk, Teun A. van (ed.) (1997): 258-284.

Faulstich Werner (1997ff.): Die Geschichte der Medien, 6 Bände (ersch. Bd. 1-4). Göttingen.

Fischer-Lichte, Erika et al. (eds.) (1995): The Touch of Difference. Tübingen.

Funk, Peter/Weiss, Hans-Jürgen (1995): "Ausländer als Medienproblem? Thematisierungseffekte der Medienberichterstattung über Ausländer, Asyl und Rechtsextremismus in Deutschland. Media Perspektiven 1: 21-29.

Galliker, Mark/Wagner Franc (1995): "Ein Kategoriensystem zur Wahrnehmung und Kodierung sprachlicher Diskriminierung". Journal für Psychologie 3: 33-43.

Geertz, Clifford (1987): Dichte Beschreibung. Beiträge zum Verstehen kultureller Systeme. Frankfurt/M. 
Genske, Dieter/Hess-Lüttich, Ernest W.B. (1999): "Conflict, Crisis, and Catastrophe. Cultural Codes and Media Management in Environmental Conflicts: the Case of Water". In: Neumann, Bernd (ed.): Dialogue Analysis and the Mass Media. Tübingen: 231-248.

Gerhard, Ute (1994): "Die Inszenierung der Katastrophe. Rassismus im Mediendiskurs". In: Rauchfleisch, Udo (ed.) (1994): 115-130.

Großklaus, Götz [2003]: "Interkulturelle Medienwissenschaft". In: Wielacher, Alois (ed.): Handbuch der Interkulturellen Germanistik. Stuttgart/Weimar.

Günthner, Susanne (1993): Diskursstrategien in der interkulturellen Kommunikation. Tübingen.

Güntürk, Reyhan (1999): "Mediennutzung der Migranten - mediale Isolation?". In: Butterwegge, Christoph et al. (eds.) (1999): 136-143.

Güntürk, Reyhan (2000): "Mediennutzung der türkischen Migranten". In: Schatz, Heribert et al. (eds.) (2000): 272-280.

Gumperz, John (1982): Discourse Strategies. Cambridge.

Hafez, Kai (1999): "Antisemitismus, Philosemitismus und Islamfeindlichkeit: ein Vergleich ethnisch-religiöser Medienbilder". In: Butterwegge, Christoph et al. (eds.) (1999): 122135.

Hansen, Klaus P. (1995): Kultur und Kulturwissenschaft. Tübingen/Basel.

Harré, Ron/Brockmeier, Jens/Mühlhäuslerk, Peter (1998): Greenspeak. A Study of Environmental Discourse. Thousand Oaks/London/New Delhi.

Hess-Lüttich, Ernest W.B. (1989): "Interkulturelle Kommunikation". In: Förster, Jürgen E. et al. (eds.): Wozu noch Germanistik? Wissenschaft - Beruf - Kulturelle Praxis. Stuttgart: 176-192.

Hess-Lüttich, Ernest W. B. (1992): "Interkulturelle Kommunikation Medienkommunikation". In: Hess-Lüttich, Ernest W. B. (1992): 23-40.

Hess-Lüttich, Ernest W. B. (ed.) (1992): Medienkultur - Kulturkonflikt. Massenmedien in der interkulturellen und internationalen Kommunikation. Opladen.

Hess-Lüttich, Ernest W. B. (1997): "Reden über die Fremden. Zum schweizerischen Migrationsdiskurs in der öffentlichen und institutionellen Kommunikation". In: Scheffer, Bernd (ed.) (1997): 193-223.

Hess-Lüttich, Ernest W. B. (2001): "Migrationsdiskurs im Film: Pepe Danquarts Schwarzfahrer und Peter von Guntens They teach us to be happy". In: Hess-Lüttich. Ernest W. B. (ed.) (2001): 209-226.

Hess-Lüttich, Ernest W. B. (ed.) (2001): Autoren, Automaten, Audiovisionen. Neue Ansätze der Medienästhetik und Telesemiotik. Wiesbaden.

Hess-Lüttich, Ernest W. B./Siegrist, Christoph/Würffel, Stefan Bodo (eds.) (1996): Fremdverstehen in Sprache, Literatur und Medien. Frankfurt/M.

Hömberg, Walter/Schlemmer, Sabine (1995): "Fremde als Objekt. Asylberichterstattung in deutschen Tageszeitungen". Media Perspektiven 1: 11-20.

Jäger, Margret (1999): "Inländische und ausländische Straftäter in deutschen Printmedien: Ergebnisse einer Untersuchung und Vorschläge zur Verbesserung der Berichterstattung". In: Butterwegge, Christoph et al. (eds.) (1999): 109-121.

Jäger, Siegfried (1993a): Kritische Diskursanalyse. Eine Einführung. Duisburg. 
Jäger, Siegfried/Link, Jürgen (eds.) (1993b): Die vierte Gewalt. Rassismus und die Medien. Duisburg.

Jäger, Siegfried (1997a): "Die Anstifter als Brandstifter? Zum Anteil der Medien an der Eskalation rassistisch motivierter Gewalt in der Bundesrepublik Deutschland". In: Scheffer, Bernd (ed.) (1997): 73-98.

Jäger, Siegfried (1997b): "Kulturkontakt - Kulturkonflikt. Ein diskursanalytisch begründeter Problemaufriß". In: Jung, Matthias et al. (eds.) (1997): 71-88.

Jäger, Siegfried (2000): "Von deutschen Einzeltätern und ausländischen banden: Medien und Straftaten". In: Schatz, Heribert et al. (eds.) (2000): 207-216.

Jandt, Fred E. (1998): Intercultural Communication. Thousand Oaks/London/New Delhi.

Janke, Hans (1987): "Vermittelte Fremde: Ausländer in den Medien". In: Bundeszentrale für Politische Bildung (ed.) (1987): 58-66.

Jarren, Otfried (1987): "Ausländerspezifische Programme neuer Anbieter - das Kabelpilotprojekt Berlin". In: Bundeszentrale für Politische Bildung (ed.) (1987): 104-111. Jung, Matthias/Wengeler, Martin/Böke, Karin (eds.) (1997): Die Sprache des Migrationsdiskurses. Das Reden über "Ausländer" in Medien, Politik und Alltag. Opladen.

Jungk, Sabine (1999): "'Mehr Farbe in die Medien' - ein Modellprojekt zur interkulturellen Öffnung der Rundfunkanstalten". In: Butterwegge, Christoph et al. (eds.) (1999): 207-227.

Koschinski, Michael (1986): Fernsehprogramme als Mittel der Integration. Eine Untersuchung interkulturelle Kommunikationsprobleme der türkischen Minderheit in der Bundesrepublik Deutschland. Essen.

Kroeber, Alfred L./Kluckhohn, Clyde (1952): Culture. A Critical Review of Concepts and Definitions. New York.

Krusche, Dietrich (1985): Literatur und Fremde. Zur Hermeneutik kulturräumlicher Distanz. München.

Kühne-Scholand, Hildegard (1987): "Die Darstellung der Ausländer im deutschen Fernsehen. Ergebnisse einer inhaltsanalytischen Untersuchung". In: Bundeszentrale für Politische Bildung (ed.) (1987): 79-84.

Küpfer, Adriano Renato (1994): "...darunter zwei Asylbewerber". Eine quantitative Inhaltsanalyse von Schweizer Tageszeitungen zur Asylthematik. Bern.

Link, Jürgen (1982): "Kollektivsymbolik und Mediendiskurse". kultuRRevolution 1: 6-21.

Luchtenberg, Sigrid (1999): Interkulturelle kommunikative Kompetenz. Opladen/Wiesbaden.

Luginbühl, Martin (1999): Gewalt im Gespräch. Verbale Gewalt in politischen Fernsehdiskussionen am Beispiel der "Arena". Bern etc.

Luhmann, Niklas (1996): Die Realität der Massenmedien. Opladen.

Maletzke, Gerhard (1996): Interkulturelle Kommunikation. Zur Interaktion zwischen Menschen verschiedener Kulturen. Opladen.

Matouschek, Bernd/Wodak, Ruth/Januschek, Franz (1995): Notwendige Maßnahmen gegen Fremde? Genese und Formen von rassistischen Diskursen der Differenz. Wien.

Meier-Braun, Karl-Heinz/Kilgus, Martin (1998): 40 Jahre "Gastarbeiter". 4. Radioforum Ausländer bei uns. Tübingen.

Merten, Klaus/Ruhrmann, Georg et al. (1986): Das Bild der Ausländer in der deutschen Presse. Ergebnisse einer systematischen Inhaltsanalyse. Frankfurt/M. 
Merten, Klaus (1987): "Das Bild der Ausländer in der deutschen Presse". In: Bundeszentrale für Politische Bildung (ed.) (1987): 69-78.

Nöth, Winfried (1996): "Ökosemiotik". Zeitschrift für Semiotik 18.1: 7-18.

Perotti, Antonio (1987): "Ausländer und Massenmedien in europäischen Ländern - ein Überblick". In: Bundeszentrale für Politische Bildung (ed.) (1987): 123-125.

Rauchfleisch, Udo (ed.) (1994): Fremd im Paradies. Migration und Rassismus. Basel.

Redder Angelika/Rehbein, Jochen (eds.) (1987): Arbeiten zur Interkulturellen Kommunikation. Osnabrück.

Rehbein, Jochen (ed.) (1985): Interkulturelle Kommunikation. Tübingen.

Reisigl, Martin/Wodak, Ruth 2000: Discourse and Discrimination. The Rhetoric of Racism and Antisemitism. London/New York.

Reisigl, Martin/Wodak, Ruth (eds.) (2000): The Semiotics of Racism. Approaches in Critical Discourse Analysis. Wien.

Ruhrmann, Georg (1993): "Die Konstruktion des 'Fremden' in den Medien". In: Jäger, Siegfried/Link, Jürgen (eds.) (1993): 190-212.

Ruhrmann, Georg (1997): "Fremde im Mediendiskurs. Ergebnisse empirischer Presse-, TVund PR-Analysen". In: Jung, Matthias et al. (eds.) (1997): 58-70.

Ruhrmann, Georg/Kollmer, Jochem (1987): Ausländerberichterstattung in der Kommune. Inhaltsanalyse Bielefelder Tageszeitungen unter besonderer Berücksichtigung ausländerfeindlicher Alltagstheorien. Opladen.

Ruhrmann, Georg (1999): "Medienberichterstattung über Ausländer: Befunde - Perspektiven Empfehlungen". In: Butterwegge, Christoph et al. (eds.) (1999): 95-108.

Sarigöz, Fatma (1999): "Die multikulturelle Gesellschaft im Spiegel der Medien". In: Butterwegge, Christoph et al. (eds.) (1999): 9-28.

Scharenberg, Albert (1999): "'Counter-Discourse' - schwarzer Nationalismus im Film". In: Butterwegge, Christoph et al. (eds.) (1999): 144-163.

Schatz, Heribert/Holz-Bacha, Christina/Nieland, Jörg-Uwe (eds.) (2000): Migranten und Medien. Neue Herausforderungen an die Integrationsfunktion von Presse und Rundfunk. Wiesbaden.

Scheffer, Bernd (ed.) (1997): Medien und Fremdenfeindlichkeit. Alltägliche Paradoxien, Dilemmata, Absurditäten und Zynismen. Opladen.

Schmidt Siegfried J. (1996): "Medienkulturwissenschaft: Interkulturelle Perspektiven". In: Wierlacher, Alois/Stötzel, Georg (ed.) (1996): Blickwinkel. München: 803-810.

Thomas, Tanja (in Vorb.): Fremde im Mediendiskurs - Diskriminierung im Gespräch. Tübingen: Diss.phil.

Tyler, Edward B. (1871): Primitive Culture. London.

Wagner, Franc/Galliker, Mark/Weimer, Daniel (1997): "Implizite sprachliche Diskriminierung von Ausländern zur Zeit der Wende". In: Jung, Matthias et al. (eds.) (1997): 230-240.

Wagner, Franc (2000): Implizite sprachliche Diskriminierung als Sprechakt. Lexikalische Indikatoren impliziter Diskriminierung in Medientexten. Tübingen. 
Weiß, Hans Jürgen et al. (1995): Gewalt von Rechts - (k)ein Fernsehthema? Zur Fernsehberichterstattung über Rechtsextremismus, Ausländer und Asyl in Deutschland. Opladen.

Wierlacher, Alois (ed.) (2000): Kulturthema Kommunikation. Möhnesee.

Winkler, Beate (1994): Was heißt denn hier fremd? Thema Ausländerfeindlichkeit. Macht und Verantwortung in den Medien. München.

Wodak, Ruth (1994): "Formen rassistischen Diskurses über Fremde". In: Brünner, Gisela/ Graefen, Gabriele (eds.) (1994): 265-284. 